\title{
Poder arcóntico y archivería virtual en el campo científico-académico. Una aproximación a prácticas en evolución y perfiles contrahegemónicos
}

Archontic power and virtual archives in the scientific-academic field. An approach to evolving practices and counterhegemonic profiles

\section{Lucia Céspedes}

Universidad Nacional de Córdoba. Centro de

Investigaciones y Estudios sobre Cultura y Sociedad

(CIECS) - CONICET, Argentina

lucia.cespedes@unc.edu.ar

(DD https://orcid.org/0000-0001-5896-3377

\section{Lisha Dávila}

Universidad Nacional de Córdoba. Centro de Estudios

Avanzados - CONICET / Universidad Nacional de Villa

María. Instituto Académico Pedagógico de Ciencias Sociales,

Argentina

lishpam@gmail.com

(iD https://orcid.org/0000-0002-0710-1475

\author{
Victor Humberto Guzmán $(\dagger)$ \\ Universidad Nacional de Córdoba. Centro de Estudios \\ Avanzados - CONICET / Universidad Nacional de Villa \\ María. Instituto Académico Pedagógico de Ciencias Sociales, \\ Argentina \\ (D) https://orcid.org/0000-0003-0302-9776
}

\section{Resumen:}

La comunicación científica entre pares no sólo ha ido evolucionando, sino que se ha transformado en un componente propulsor de los cambios de paradigma en cuanto a la práctica e institucionalidad de las ciencias. Movidas por la importancia del registro y la conservación de sus textos, las ciencias se fueron dotando de un orden archivístico propio donde ciertos agentes se tornaron los arcontes del campo y la publicación científica se fue convirtiendo en un mercado cada vez más concentrado. Si bien la revolución digital y las posibilidades inauguradas por las nuevas tecnologías de información y comunicación fortalecieron estos modelos, se inauguraron al mismo tiempo nuevas prácticas de publicación y archivo que pueden considerarse como contrarias al modelo comercial hegemónico. En este trabajo nos proponemos dar cuenta de la evolución de las prácticas de archivería científica a fin de identificar algunas de las características que suponen el ejercicio de distintas modalidades de poder arcóntico hegemónico en el campo de producción y circulación del conocimiento científico-académico. A partir de allí, reflexionamos acerca de la naturaleza y potencialidades de la archivería virtual, las diversas modalidades de acceso abierto y el surgimiento de prácticas con ciertos rasgos contrahegemónicos, capaces de introducir alteraciones, ruptura de modelos dominantes, desorden, e incluso la posibilidad de un nuevo orden de archivo de acceso al conocimiento y de poder de acción de los arcontes.

Palabras clave: Archivo virtual, Publicación científica, Revistas académicas, Producción y circulación de conocimiento, Acceso abierto, Arcontes.

\section{Abstract:}

Scientific communication among peers evolved over time to become a key propeller of paradigm shifts as regards scientific practice and institutionalization. Spurred by the importance of registration and conservation of their texts, sciences developed an archival order of their own where certain agents became the field's archons, and scientific publishing turned into an increasingly concentrated market. While the digital revolution and the possibilities brought about by information technologies strengthened 
this model, new publishing and archiving practices (which can be considered as opposing the hegemonic, commercial model) were also inaugurated. In this paper we seek to account for the evolution of scientific archives in order to identify some of the traits associated to different modalities of archontic power in the field of scientific-academic knowledge production and circulation. We then reflect on the nature and potentialities of virtual archives, the diversity of open access models, and the emergence of practices with counterhegemonic traits, capable of introducing alterations, breaking with dominant models, and even suggesting a new order of knowledge archive, accessibility, and archontic power.

KEYWORDS: Virtual archive, Scientific publishing, Academic journals, Production and circulation of knowledge, Open access, Archons.

\section{Este trabajo está dedicado a la memoria de nuestro coautor, amigo y compañero, Víctor Humberto Guzmán, con quien sembramos algunas semillas de su eterno limonero.}

\section{INTRODUCCIÓN}

Entre los procesos que trajo aparejada la inauguración de las ciencias modernas, nos interesa hacer énfasis en la importancia de la acumulación del conjunto de conocimientos como factor característico. Si bien la ciencia se realiza (produce) en los laboratorios, las oficinas, o en el trabajo de campo, también es cierto que el conocimiento generado no se queda allí. En este proceso -e incluso ya desde la etapa de producción- la comunicación científica juega un rol importante, más aún al momento de hacer público el conocimiento.

La comunicación entre pares no sólo ha evolucionado con el tiempo, sino que se ha transformado en el componente propulsor que da cuenta de la acumulación del conocimiento como base del sistema científico. Los textos (como principal código), en tanto documentos, deben ser conservados bajo cierto orden $\mathrm{u}$ organización. Así, la bibliotecología, la documentación y la archivería no sólo fueron adquiriendo entidad propia como disciplinas científicas, sino que se tornaron herramientas indispensables para el desarrollo de las ciencias en general. Por ello, toda pregunta por las lógicas actuales de producción, circulación, comunicación y apropiación social del conocimiento académico y científico-tecnológico necesariamente debe tomar en cuenta la dimensión archivística de esta actividad.

Es, sobre todo, a partir de la consolidación (durante el siglo XX) del artículo y la revista especializada como los formatos preferidos de la comunicación endógena intra e interdisciplinar (Verón, 1998) que las publicaciones científico-académicas comienzan a proliferar. Ya en la década de los ‘60, Derek De Solla Price (1965) estipuló, en un estudio hoy clásico, que la cantidad de revistas académicas y trabajadores científicos se había ido duplicando aproximadamente cada quince años entre 1660 y 1960 (en estudios más recientes, la duplicación de la cantidad de artículos publicados desde 1985 se estableció en 24 años (Jinha, 2010).

A lo largo de ese proceso, y acompañando los cambios de paradigma en cuanto a la práctica e institucionalidad de las ciencias, se fueron delineando e imponiendo determinadas modalidades de archivo y, en consecuencia, de acceso al conocimiento. Es decir, las ciencias se fueron dotando de un orden archivístico propio donde ciertos agentes se tornaron los arcontes del campo. Esta noción empleada por Jacques Derrida (1997) hace referencia a los arcontes como aquellos agentes que tienen el deber de resguardar los archivos, pero también el derecho hermenéutico de interpretarlos. Esta potestad sobre los archivos constituye el poder arcóntico, que, como todo poder, puede convertirse en hegemónico.

La importancia de la acumulación de conocimiento científico y el rédito generado por el ejercicio del archivo y la documentación impulsó el surgimiento de conglomerados de empresas de información científica y grupos editoriales que fueron constituyendo un orden informacional propietario, cerrado, exclusivo y excluyente en términos económicos y simbólicos, a la vez que la edición y publicación de revistas científicas se fue convirtiendo en un mercado cada vez más dominado por unos pocos agentes. Hacia fines del siglo XX, la revolución digital y las posibilidades inauguradas por las nuevas tecnologías de información 
y comunicación introdujeron cambios cualitativos y cuantitativos en el orden archivístico que, hasta el momento, se constreñía en los límites físicos del papel impreso. Si la digitalización mejoró el acceso y facilitó la búsqueda y las vinculaciones entre artículos y revistas, el formato y las gramáticas de lectura no se modificaron sustancialmente. Lo que sí cambió fue el aspecto económico de la edición y la publicación académica, devenida un mercado altamente rentable y de estructura crecientemente oligopólica (Larivière, Haustein y Mongeon, 2015). En un campo donde las revistas cambian de editorial al ser compradas, y las editoriales también se compran o fusionan con sus aliados (o competidores) comerciales, la concentración del mercado se refleja en el porcentaje de revistas, artículos y citas pertenecientes a los cinco grupos-conglomerados editoriales más grandes: RELX (anteriormente Reed-Elsevier), Wiley-Blackwell, Springer, Sage Publications, y Taylor \& Francis. Tanto para las ciencias naturales y biomédicas como para las sociales y humanas, estas cinco editoriales dan cuenta de más de la mitad de las revistas académicas en la actualidad (Larivière et al., 2015).

A la vez, la comunicación científica a través de la web inauguró nuevas prácticas de publicación y archivo, algunas de las cuales pueden considerarse como contrarias a este modelo comercial hegemónico. Nos referimos a las diversas modalidades de Acceso Abierto (AA), al surgimiento de redes sociales académicas, a los repositorios institucionales y de preprints, e incluso a sitios web que, operando al margen de los órdenes restrictivos de propiedad intelectual, pueden considerarse de "piratería" editorial y científica.

Con estas consideraciones en mente, en este trabajo nos proponemos dar cuenta de la evolución de las prácticas de archivería científica a fin de identificar algunas de las características que suponen el ejercicio de distintas modalidades de poder arcóntico hegemónico en el campo de producción y circulación del conocimiento científico-académico. A partir de allí, reflexionamos acerca de la naturaleza y potencialidades de la archivería virtual en procesos de apropiación social del conocimiento y en prácticas contrahegemónicas emergentes, capaces de introducir alteraciones, ruptura de modelos dominantes, desorden, e incluso la posibilidad de proyectar un nuevo orden de archivo, de acceso al conocimiento y de arcontes.

\section{2. ¿Cómo DEFINIR UN ARCONTE PARA LAS CIENCIAS?}

Una de las tesis que sostendremos a lo largo de este artículo es que, desde sus comienzos, la ciencia como práctica humana ha tenido un carácter público (o tendiente a lo público). Posiblemente en la actualidad relacionemos esta noción con el origen de financiamiento de las actividades científico-tecnológicas (proveniente del sector privado, identificado con la empresa o el filántropo particular versus proveniente del sector público, identificado con el Estado), pero en una concepción más amplia, es el propio conocimiento científico lo que se construye de forma intersubjetiva. Si en los inicios estos intercambios se daban a través de la correspondencia entre los primeros "hombres de ciencia" o "filósofos naturales", la institucionalización de la práctica científica fue consolidando mecanismos formales de circular y compartir las ideas como condición de posibilidad para el avance del conocimiento.

Esta idea difícilmente pueda ser novedosa: el comunismo entendido como el ideal de colaboración social entre científicos, el conocimiento como propiedad comunitaria y herencia común, y su comunicación como imperativo, forman parte de los componentes del ethos mertoniano. Podemos entonces ir un paso más lejos: la ciencia siempre se ha hecho para serpública, y, para eso, se publica. Como rezaba en su momento el slogan del portal RedALyC, "la ciencia que no se ve no existe". Permitámonos innovar jugando a propósito con las diferentes acepciones del término publicar y sus derivados, ya que la polisemia intrínseca de esta palabra brinda más de una capa de interpretación al fenómeno bajo análisis. Publicar puede querer decir, en su sentido más directo, generar y difundir copias de un texto por medios físicos o electrónicos. A la vez, remite tanto a la idea de develar algo que estaba oculto como al hecho de difundirlo para hacerlo públicamente conocido, darle publicidad. Es decir, en el mismo movimiento en que un texto es publicado, se da a conocer a un público determinado. 
En la actualidad, la ciencia efectivamente se hace pública (al menos dentro del más o menos restringido ámbito de la comunidad de pares) mediante la publicación de textos de características muy particulares, primordialmente, el hegemónico artículo científico o paper (de dominio casi absoluto en las ciencias físicas, exactas y naturales, mientras que en las ciencias sociales y humanidades el libro sigue siendo un estilo de publicación importante, sin contar otras formas de comunicación científica marcadas por la oralidad). El artículo es la condensación de un proceso de investigación, que reúne aquello que sus autores ponen a consideración de sus colegas: un resultado, un aporte novedoso al acervo común de conocimiento científico. Para Rainer Hamel (2013), ese resultado se identifica justamente con "la publicación objetivada en la escritura y difundida en órganos legitimados por el propio campo", cuya producción implica un "procedimiento ideológico típico de las ciencias que esconde a los actores y los procesos mismos detrás de una pantalla - la publicación científica" (Hamel, 2013, p. 350). Ampliaremos la discusión acerca de los espacios de publicación más adelante; por el momento, baste decir que consideramos a los artículos como la forma textual que adopta un resultado de investigación (en tanto culminación o avance de un proceso) para ser puesto en circulación de manera legítima y apropiado por los agentes dentro del campo.

Esto nos habilita a pensar que, a través de la acción de publicación, el texto (la ciencia) queda al mismo tiempo archivado, es decir, se inscribe como huella de un proceso creativo-intelectual en una determinada superficie de registro. Después de todo, una de las premisas básicas de la práctica científica es que todo resultado debe ser replicable, y para ello se requiere su almacenamiento de manera accesible al resto de la comunidad de expertos. Al respecto, si desde una perspectiva orgánica del archivo "el principio de procedencia es un procedimiento sistemático de identificación, ordenamiento y conservación de los registros a partir de su cuerpo o institución de origen" (Tello, 2018a, p. 46, cursiva en el original), en la CyT, los criterios y procedimientos de orden se relacionan con la prioridad más que con la procedencia: quien publique primero será recordado como el descubridor; los que vengan después, serán las réplicas y confirmaciones de ese trabajo pionero.

Llegamos entonces a la premisa de que, en la ciencia, publicar y archivar son acciones simultáneas. ${ }^{1}$ Como dice Jean-Claude Guédon (2019), un artículo obliga a ejecutar las funciones de publicación, preservación y diseminación en un mismo momento. $\mathrm{Y}$ tal como ocurre con publicar, archivar admite diversas interpretaciones ya que, siguiendo la postura derrideana de Andrés Tello (2018a), la noción misma de archivo no remite a lo "ya sido" sino a una promesa abierta de decodificación de su significado a futuro. Esto implica la irreductibilidad del archivo a identificaciones o clasificaciones fijas y permanentes. En el caso de la ciencia, ¿cómo clasificar el contenido de las labores archivadas, es decir, el conocimiento científico, en un momento en que las fronteras disciplinares están estallando y el trabajo es cada vez más multi, inter, transdisciplinar?Las superficies y los soportes virtuales habilitados por las nuevas tecnologías desafían los modos y definiciones tradicionales de archivo. Todo esto dificulta el encasillamiento de esos materiales en categorías textuales, y por ende archivísticas, fijas.

Frente a esta multiplicidad, tomamos de Tello (2018a) una definición operativa de archivo como una memoria encarnada en una superficie o soporte, intrínsecamente atravesada por distintos campos de fuerza y tensiones. De esto se desprende el vínculo entre archivo y poder, o, al decir del autor, "una cierta economía del poder, entendida como el despliegue de una administración particular de los registros, de las huellas de los acontecimientos y las experiencias inscritas de un modo u otro sobre la superficie de un soporte" (Tello, 2018a, p. 54). El poder con relación al archivo se condensa en la figura del arconte, aquel que dispone de los procedimientos de selección, supresión, prohibición, control, y represión sobre los registros. El autor sostiene que "el poder arcóntico despliega toda una economía de gestión de los registros en los espacios y soportes de almacenamiento, ejerciendo un monopolio o una capitalización sobre aquellas huellas instituidas o reconocidas como legítimas por su ley" (Tello, 2018b, p. 220).

Este despliegue instaura patrones de organización de los registros situados en un espacio-tiempo determinado. Las huellas efectivamente registradas son aquellas sobrevivientes de permanentes batallas por 
la producción de ese ordenamiento, de esa organización de discursos y cuerpos sociales en archivos. El resto del proceso integral de hacer ciencia, que queda fuera del relato lineal del paper, sucumbe a la violencia archivadora del archivo mismo, a su pulsión de destruir sus propias huellas, como señala Derrida (1997). Dentro del campo científico, factores estructurales como los criterios de evaluación basados en el recuento de publicaciones y citas (Vasen y Lujano Vilchis, 2017), y la elaboración de rankings que miden la "excelencia científica" de las instituciones (ver Vessuri, Guédon y Cetto, 2014, para una discusión de este término) con valores numéricos y métricas diversas, no sólo hacen, como ya mencionamos, que la actividad científica se identifique únicamente con su resultado final, sino que fomentan este impulso de publicación por parte de los investigadores (mientras que la faceta del archivo queda en manos de los arcontes, como desarrollaremos más adelante).

Ciertamente, los criterios de evaluación institucionales y el sistema de premios-castigos que establecen son uno de los principales factores que orientan las estrategias de los agentes en el campo científico-académico, tanto en cuanto a las prácticas de edición y publicación de revistas como a la producción de conocimiento en sí (ver Vasen y Lujano Vilchis, 2017, para un análisis comparativo de los sistemas nacionales de evaluación de revistas en países latinoamericanos). En términos de Tello (2018b, p. 229), se pueden interpretar como técnicas modeladoras de conductas que permiten "maximizar el rendimiento de los cuerpos individuales en pos de la aceleración industrial del proceso de acumulación capitalista”. En el marco interpretativo del capitalismo arcóntico propuesto por el autor, un agente productor de conocimiento científico se define y vale por lo que dicta su corpus archivístico. Esta pulsión archivística, traducida en el ámbito de la práctica científica como el conocido adagio de "publica o perece", termina generando el efecto de disociar a los productos del campo, las "verdades científicas" plasmadas en textos, de sus condiciones sociales de producción (Bourdieu, 1994).

Si consideramos que toda publicación involucra una forma de archivo, la práctica de archivo sería constitutiva de las ciencias. Desde el origen de lo que en Occidente definimos como ciencia, esta ha estado íntimamente ligada a la posibilidad de conservación del conocimiento para su transmisión en el tiempo, pasando por diversas técnicas "de archivación, de impresión, de inscripción, de reproducción, de formalización, de cifrado y traducción de marcas" (Derrida, 1997, p. 22), con sus consiguientes "transformaciones jurídicas y, por tanto, políticas. Éstas afectan nada menos que al derecho de propiedad, al derecho de publicar y de reproducir" (Derrida, 1997, p. 25). Con la institucionalización y profesionalización de la práctica científica, el campo se fue dando de sus propios instrumentos de archivo, hasta llegar a la revista académica actual como el soporte canónico y más extendido.

Consecuentemente, sobre las revistas recaería la potestad de vigilar el depósito de documentos y la autoridad sobre su conservación, circulación y acceso. Esto constituye la dimensión de poder político inherente a todo archivo, derivada de su finitud: la imposibilidad física de publicar/archivar todo necesariamente conlleva un proceso de selección. Las ciencias no son la excepción: claramente, no todo lo que se produce llega a publicarse (sólo aquello que pase por un referato preferentemente de doble ciego), y no todo lo que se publica se valora de la misma manera. Piénsese en cómo las revistas más prestigiosas dentro del campo científico se jactan de sus altas tasas de rechazo de artículos para consolidar una imagen de extrema rigurosidad en la selección del contenido a publicar, el cual, en función de haber superado esos exigentes requisitos, se juzga trabajo "de excelencia".

En este sentido, el arconte, al definir los rasgos que debe tener un texto publicable-archivable, también tiene el poder de direccionar la investigación misma. Es decir, así como para Michel Foucault (2002) el archivo establece un horizonte discursivo del orden de lo decible en la sociedad, los arcontes hegemónicos de la ciencia, mediante la imposición de sus criterios de publicación-archivo, contribuyen a definir los horizontes de $l o$ investigable en un determinado momento. Después de todo, ¿ por qué alguien se molestaría en invertir tiempo, esfuerzo y recursos en investigar un tema que no tuviera perspectivas de ser publicado? ¿Quién querría quedar afuera de la conversación en torno al conocimiento que se juzga valioso y susceptible de ser conservado? Para 
Derrida (1997) esto es clave, ya que no hay archivo sin exterioridad. Preguntarse dónde comienza el afuera es la cuestión del archivo, pues implica preguntarse por la frontera, qué se decide incluir y qué no, y, nuevamente, quién o quiénes detentan esa capacidad de decisión. Los arcontes de la ciencia, en definitiva, tienen un poder nada desdeñable para definir qué modos de conocimiento se consideran ciencia buena, sólida y legítima, y cuáles no.

A esta altura de la discusión, al lector familiarizado con las prácticas de publicación dentro del campo científico comenzarán a resonarle ciertos candidatos al rol de arcontes en este ámbito. Si en la sociedad de control este papel recae en plataformas como Facebook o Google, en sujetos como Mark Zuckerberg, Larry Page y Serguéi Brin (Tello, 2018b), en la ciencia mainstream internacional los arcontes serían conglomerados editoriales como Elsevier o SAGE, y bases de datos como Scopus o Web of Science. Son, en gran medida, los que concentran y ejercen el poder arcóntico al establecer las reglas del juego en los circuitos de publicación y circulación del conocimiento de mayor prestigio y centralidad. ${ }^{2}$

Sin embargo, estos no son ni han sido los únicos arcontes de las ciencias. A lo largo de la historia de producción, circulación y apropiación del conocimiento científico, el poder arcóntico ha sido ejercido bajo diferentes modalidades. Además, en cuanto nos apartamos del modelo editorial transnacional, corporativo, comercial y privado, aparecen otros agentes y otras modalidades de poder arcóntico vinculadas a otras formas de publicar y archivar. De su descripción y caracterización nos ocuparemos en los apartados siguientes.

\section{Evolución histórica de la CIRCULACión (PÚBLICA) DEL CONOCIMIENTO CIENTÍFICO COMO CORRELATO DE LA VISIÓN CIENTÍFICA DEL MUNDO}

El decurso del tiempo no sólo modifica la concepción, producción y validación del conocimiento científico, sino también su circulación y comunicación en tanto práctica humana. Por ello, con la transformación de la ciencia también se producen cambios en su estructura comunicacional.

Estas afirmaciones se hacen evidentes, por ejemplo, ante la obertura de la época moderna, comprendida entre los siglos XVI y XVIII, que se constituyó en un "arco temporal" (Polino, 2014) que puso en evidencia no sólo nuevas estructuras económicas, políticas, religiosas, culturales y mentales, sino también transformaciones en la visión del universo, del hombre y de la ciencia. En este contexto socio-económico también es posible identificar ciertos factores determinantes que marcaron el desarrollo de la comunicación de la ciencia moderna: la nueva visión caracterizada a partir de la gestación de la revolución científica; el desarrollo de la imprenta (que, como indica Rossi (2000) fue una innovación técnica que no sólo facilitó el acceso al conocimiento, sino también transformó la comunicación en un valor), y el proceso de institucionalización de la ciencia moderna, con la aparición de las sociedades científicas y un nuevo concepto de comunicación basado en la publicación impresa de journals. Fue así como el sistema de correspondencia de manuscritos, utilizado por la comunidad científica e intelectual, conocida como République des Lettres, se fue transformando progresivamente.

$\mathrm{Al}$ contenido intelectual se añadieron gradualmente comentarios, evaluaciones y juicios que se desarrollaron durante su tiempo en un método completo de expresión crítica. [...] Pero la producción creciente de materiales no era compatible con la capacidad de estos "hombres de letras" para escribir cartas. La solución para tal dilema surgió en la forma de la prensa (Sabbatini, 1999, pp. 5-6).

La nueva visión científica requirió de la prensa para su comunicación, y con esta tecnología, como señala Marcelo Sabbatini (1999), la ciencia no sólo dejó la privacidad de las cartas y pasó a la exhibición pública, sino que también adquirió mayor legitimidad y el privilegio de la conservación. Aunque los objetivos de esta apertura no sólo se centraban en la comunicación escrita a través de libros, cartas o diálogos, sino también en la invención de espacios como gabinetes, teatros y conferencias (Polino, 2014), hubo una mayor significación de iniciativas tales como las revistas científicas y las publicaciones académicas. 
Fueron las academias y sociedades científicas -creadas al margen de las universidades- quienes convirtieron la ciencia en una institución y fijaron entre sus principales objetivos la comunicación y transmisión de los resultados obtenidos y de los métodos utilizados en los experimentos. La incorporación de la publicación como parte del proceso científico realzó la importancia y legitimidad de la ciencia y puso de relieve la trascendencia de la comunicación científica. Esta transformación se sustenta, sobre todo, a partir la publicación de los trabajos y debates que dieron paso y origen a las revistas periódicas.

En 1665, [se produjo] la aparición del Journal des Sçavans, en Francia, seguido por las [Philosophical] Transactions of the Royal Society of London, en el mismo año. Luego, las publicaciones aparecieron en Italia, con la emergencia de Litteratti de Italia en 1668 y en Alemania, con la Miscellanea Curiosa, en 1670 (Sabbatini, 1999, pp. 6-7).

Las revistas científicas no sólo se convirtieron en transmisoras de información, sino que posibilitaron la generación de un sistema de protocolos, verificación, reproductibilidad, reconocimiento, revisión, conservación, documentación y archivo. Como señala Guédon (1996), comenzaron a construir jurisprudencia, memoria y registro de elementos y formas. Primero, a partir de la puesta en común de las correspondencias, reconvertidas en debates; posteriormente con descripciones y observaciones que intentaban dar cuenta del proceso; y más adelante desde una estructura organizativa denominada IMMRyD (Introducción, Materiales y Métodos, Resultados y Discusión) -que da origen a lo que hoy conocemos como artículo científico o paper. Como remarcan Sara Mendoza y Tatiana Paravic (2006), se considera que fue Philosophical transactions la primera revista en iniciar, en 1752, el sistema de revisión por pares, para determinar si los artículos que recibían merecían ser publicados.

Durante el siglo XIX, la ciencia y las prácticas de investigación comienzan a ser incorporadas en las universidades dando paso a la fase profesionalizadora de la ciencia. Este proceso coincidió además con la imposición de nuevos paradigmas científicos para la interpretación de la naturaleza (Polino, 2014) y vino acompañado con el crecimiento de las comunidades científicas y de las publicaciones periódicas especializadas. Cabe destacar que, durante este vertiginoso desarrollo de la ciencia y su comunicación, el principal objetivo era el intercambio y puesta en común del conocimiento, no el lucro ni rédito comercial. Si bien, como señala Ysabel Briceño (2013), algunas publicaciones se vendían al público, casi siempre un miembro de una sociedad recibía sus publicaciones gratuitas a cambio de su suscripción y se realizaban intercambios con otros ejemplares entre personas e instituciones. Fue así que las sociedades, instituciones y observatorios desarrollaban sus colecciones bibliográficas de revistas especializadas, aunque todavía las ediciones eran limitadas.

Mencionar a la comunidad científica como prosumidora y arconte tiene relación con esta descripción en la cual damos cuenta del rol de los intelectuales y científicos como gestores, productores y consumidores de las publicaciones científicas, pero además de su tarea como guardianes responsables de la seguridad física del depósito y el cuidado de estos documentos, junto al derecho de ejercer la competencia hermenéutica sobre ellos. Si bien durante algunos años fueron las sociedades científicas y las propias universidades quienes controlaron y dirigieron la publicación de trabajos académicos y científicos, desde pequeños grupos editoriales y organizaciones sin ánimo de lucro; en la segunda mitad del siglo XX las instituciones educativas empezaron a desvincularse de ese rol ante el progresivo avance de editoriales comerciales que vieron en la publicación científica una oportunidad para hacer negocios (Fresco, 2013).

Esta progresión hizo cada vez más evidente la importancia asignada al sistema de protocolos que se habían definido con las primeras revistas científicas, y algunos se potenciaron y modernizaron como mecanismos de validación del conocimiento. Entre ellos se destaca el proceso de revisión por pares o peer review con la instauración de revisores externos al propio equipo editorial y anónimos para los autores (Briceño, 2013). Difundir los conocimientos sometidos a evaluación garantizaba transparencia, calidad científica y reconocimiento -cualidades que permanecen hasta ahora. El crédito otorgado a los descubridores y científicos, sumado al aumento de publicaciones, llevó a que los sistemas de validación tomen como dato 
relevante el estudio de las citas a los artículos ya publicados y este dato se convirtió también en una forma de entender el desempeño científico (Fresco, 2013). A partir de este momento, tal como reflexiona Guédon (2019), la naturaleza de las revistas se transformó: dejaron de tener como principal función la difusión de nuevos descubrimientos, y pasaron a crear un sistema de prestigio, organizado a través de métricas como el factor de impacto.

Sumado a ello, en esta nueva etapa de la comunicación científica y ante el aumento de las publicaciones acumuladas en un volumen de información inmanejable, junto a la necesidad de que el contenido de estos documentos pueda recuperarse para nuevas investigaciones, comprobaciones o aplicaciones prácticas, fue menester la creación de archivos que superaran las antiguas colecciones bibliográficas y recopilaran, organizaran y consignaran la mayor cantidad de escritos y conocimientos. Así apareció lo que hoy entendemos por colecciones de revistas y bibliotecas científicas. Muy pronto en esta tarea surgieron intereses comerciales que convirtieron el archivo y la acumulación de saberes en una oportunidad de negocio.

Las editoriales universitarias y asociaciones científicas se vieron desbordadas e incapaces de competir con los nuevos actores, que ofrecían a los investigadores no sólo un cauce de comunicación, sino servicios adicionales como la distribución rápida y puntual, contenidos de calidad, etc., por lo que muchas de ellas acabaron por ceder la gestión de sus publicaciones. (Fresco, 2013, pp.15-16).

Estos cambios y acontecimientos, lejos de generar una apertura, mayor distribución y puesta en común de las revistas científicas, sentaron las bases del manejo oligopólico y comercial de las publicaciones. De esta manera, como apunta Ana María Fresco (2013), en los años '80 las publicaciones científicas fueron concentrándose en manos de empresas privadas, que controlan y gestionan la mayoría de las revistas académicas, incluyendo las creadas por instituciones educativas. El paso siguiente fue la creación de grandes conglomerados por parte las principales empresas editoriales que ya habían dominado el mercado. El poder generado por estas grandes compañías, convertidas en los nuevos arcontes del conocimiento, les otorgó la posibilidad de fijar pautas en las distintas etapas del proceso de comunicación, sobre todo relacionadas con la distribución y el acceso, que eran las áreas desde donde mayor rédito económico podían obtener.

\section{Corporativos O ACCESIBLES, LAS MODALIDADES DEL PODER ARCóNTICO EN LA ACADEMIA}

El sistema de publicaciones científicas sufrió nuevas alteraciones a partir de los años ' 90 con el desarrollo e influencia de las nuevas tecnologías de comunicación y, en particular de la llegada de Internet. El beneficio de las TICs sería difícil de discutir si no consideráramos que también forma parte importante del capitalismo cognitivo, caracterizado por el desarrollo cuantitativo y cualitativo de las diferentes formas de capital intangible y los componentes inmateriales insertos en los diferentes productos y procesos de producción. Así como con la imprenta, Internet facilitó el desarrollo de una nueva etapa de comunicación y distribución del conocimiento. Las revistas científicas, ahora electrónicas, posibilitaron la restitución del rol de prosumidor, ${ }^{3}$ y arconte a los científicos y autores, que además de crear pueden compartir información y a la vez ser editores, revisores y distribuidores, por lo que la mediación de editores comerciales se puso en tensión. Paulatinamente se fueron articulando nuevos modelos de comunicación científica al margen de las editoriales comerciales y se empezaron a plantear vías alternativas para mejorar el acceso y la difusión de los conocimientos científicos.

El surgimiento de alternativas a la publicación académica "convencional", personificado en la creación de repositorios, el avance del movimiento Open Access y la progresiva adopción de las tecnologías de la Web 2.0, ha permitido recuperar la competencia en un mercado cuyo principal activo -la información científica- estaba siendo monopolizado (Fresco, 2013, p. 24).

Estas consideraciones llevan a pensar en el paso del archivo científico analógico al virtual como un propulsor de prácticas que se perfilaban como contrahegemónicas, en oposición al modelo comercial sustentado en el ejercicio de poder arcóntico sobre las publicaciones y archivos físicos. Pero, si bien la 
digitalización creciente del conocimiento convertido en información da paso a la expansión de los archivos virtuales y facilita el acceso a mayor cantidad de personas, también constituyó un ambiente propicio para que se acentúe su comercialización y apropiación privada. Por lo tanto, en el actual sistema global de puesta en disposición pública del conocimiento académico encontramos que conviven diversas modalidades de ejercicio del poder arcóntico.

Para ordenar la conceptualización del estado del campo, decidimos desarrollar dos grandes categorías de modalidades para este fenómeno. Dentro de una primera tipología de arcontes, que definiremos como corporativos, podemos ubicar por un lado a las editoriales y revistas comerciales y, por otro, a los índices de impacto. Dentro de una segunda categoría, que definiremos como accesibles, encontramos revistas y plataformas no comerciales. Estas dos categorías comparten como fin la legitimación del conocimiento científico como tal. Cabe aclarar que no aspiramos a brindar un listado exhaustivo de agentes identificados como corporativos o accesibles, pero sí un pantallazo del estado del campo y algunas de las principales iniciativas de publicación y archivo que pueden ubicarse en una u otra categoría.

Si bien encontramos un importante caudal de materiales disponibles en acceso abierto por fuera de estas dos modalidades, resolvemos nada más que mencionar este punto ya que entendemos que el poder de definición de los límites acerca de lo que es o no conocimiento científico se sostiene en las modalidades corporativa y accesibles. Puntualmente pensamos en lo que se definió como la "vía negra" del acceso abierto (Björk, 2017) y las plataformas privadas de autoarchivo. Esta vía negra es una de las modalidades de la puesta en común de modo abierto que brinda, a partir de aplicaciones o bases de datos de consulta online, acceso a artículos que se presentan como de acceso restringido, sujeto a pago previo o suscripción personal o institucional. Un caso paradigmático de base de datos no autorizadas es el portal Sci-Hub que otorga acceso a más del 65\% de los artículos de acceso restringido (Himmelstein et al., 2018). Otro ejemplo de servicios bajo esta modalidad, esta vez de carácter autorizado, es la aplicación Unpaywall. Esta se puede instalar como extensión de navegadores e informa, en el momento en el que se lee un resumen de un artículo de acceso restringido, si se encuentra disponible en alguna plataforma en la web y brinda un vínculo de acceso en los casos en que aparece un hallazgo.

Tanto la modalidad arcóntica corporativa como la accesible están constituidas por distintas dimensiones: la calidad o pertinencia del trabajo académico, su accesibilidad pública y su impacto. Respecto de la calidad, los dos modelos arcónticos utilizan criterios similares. La herramienta más importante con la que cuentan, en este sentido, es el de la revisión por pares para el caso de los artículos académicos, los comités editoriales para libros y la posibilidad de rendición de cuentas para la dimensión del autoarchivo.

En el caso de arcontes corporativos, algunas de las bases de datos bibliográficas más importantes son Scopus (perteneciente a la empresa Elsevier, del grupo RELX), Ebsco (perteneciente a Ebsco Publishing Inc.) y Web of Science (perteneciente a la empresa Clarivate Analytics). A su vez, algunas de las editoriales más importantes en esta modalidad son Sage Publishing (con más de mil revistas); Taylor \& Francis Group (perteneciente a la multinacional Informa PLC y que cuenta con más de 2600 revistas); John Wiley \& Sons, Inc. (con más de 1600 revistas) o Elsevier (con más de 2500 revistas).

Las bases de datos indicadas proponen ciertos criterios básicos para incorporar materiales académicos. Entre otros, incluyen la explicitación de política de revisión de los artículos mediante la revisión por pares, la regularidad en la publicación, la demostración de la incorporación de contenido relevante de su campo de especialidad y la legibilidad para una audiencia global (idioma de los artículos o resúmenes). Pero la particularidad de estas bases es que, además de estos criterios básicos, incorporan el impacto de las publicaciones. Lo importante en este punto es que este criterio para la inclusión de la publicación en la base no está relacionado con la calidad sino con la performance de sus artículos (básicamente, la cantidad y tipo de citas que reciben e incorporan funcionan como valor de la "calidad" de los trabajos). Mientras que para Ebsco y $W O S$ es un criterio previo a la incorporación en la base la presencia de citas a otras revistas ya incorporadas 
en $E b s c o$ o $W O S$, Scopus evalúa el impacto una vez que ya se encuentra incorporada la publicación en la base. Si no mejora su rendimiento luego de una revisión, esta publicación es retirada.

Respecto de la dimensión de impacto, en los arcontes corporativos encontramos dos empresas que dominan globalmente el campo. Por un lado, Clarivate Analytics, a través del Instituto para la Información Científica (ISI, por sus siglas en inglés). ISI produce el SCIE (Science Citation Index Expanded); el SSCI (Social Sciences Citation Index); el AHCI (Arts \& Humanities Citation Index); el ESCI (Emerging Sources Citation Index); el BCI (Book Citation Index); el CPCI (Conference Proceedings Citation Index); el DCI (Data Citation Index); el DPCI (Derwent Patents Citation Index); el BIOSIS Citation Index; el Russian Science Citation Index; el Chinese Science Citation Index y el SciELO Citation Index creado a partir de un acuerdo de Scielo con WOS en 2014 (Packer, 2014).

Por otro lado, Scopus produce métricas de impacto propias y, en cooperación con otras empresas, diversos índices de impacto. El índice de Scopus es CiteScore Metrics y cuenta con otros en cooperación con empresas como, por ejemplo, Scimago, que nace como un grupo de investigación de la Universidad de Granada y que tenía como objeto las publicaciones científicas y la visibilidad regional y global de esta práctica. Hacia el año 2008, Scimago se asocia con Scopus y se constituye como empresa que brinda el servicio de la lectura y conformación, a partir de la base de datos Scopus, de índices de impacto de publicaciones e instituciones académicas. Se crean en este marco los índices SJR (SCImago Journal \& Country Rank) y el SIR (Scimago Institutions Rankings). Otro ejemplo es el acuerdo que Scopus firma con la Universidad de Leiden, a partir del que se produce el SNIP (Source Normalized Impact per Paper). Vemos en este sentido una circularidad para la reproducción de la legitimidad de los arcontes corporativos, al imponerse globalmente como criterio de calidad dimensiones del proceso creativo científico ajenos a la propia práctica científico-académica.

Así, el dominio de las métricas corporativas como modo de evaluar la calidad de los trabajos generó la preocupación y reacción de esa otra configuración académica que reniega de la lógica corporativa para la definición de estos asuntos y que avanzan de manera sostenida hacia una lógica arcóntica distinta. Dentro de este campo encontramos, por ejemplo, los manifiestos de San Francisco sobre la Evaluación de la Investigación y el de Leiden sobre Indicadores de Investigación. En este sentido, señala Dominique Babini (2019, p. 120):

\footnotetext{
...se observa una tendencia a la revisión de los procesos de evaluación y sus indicadores, y al seguimiento de las recomendaciones de las principales declaraciones de la comunidad científica y académica en relación a los procesos de evaluación. Dos de las principales declaraciones internacionales que inspiran la revisión de los procesos de evaluación, y la incorporación de nuevos indicadores, son las declaraciones DORA y Leiden.
}

Estas manifestaciones se asientan sobre la estela del movimiento por el AA que, si bien encuentra raíces en la década del '70 y de manera más clara a principios de los años '90 (Moore, 2019), se puede decir que su nacimiento canónico se da con la Iniciativa por el Acceso Abierto de Budapest en 2002, la declaración de Bethesda, y la de Berlín de 2003. Estos tres momentos establecieron un marco general de principios alternativos a la lógica arcóntica corporativa de la comunicación científica. A partir de este punto se desarrollaron y desarrollan múltiples herramientas y estrategias para la implementación de políticas y prácticas orientadas a una comunicación abierta y libre del conocimiento científico académico. Con ese impulso inicial se lanza, en 2003, el Directory of Open Access Journals (DOAJ), una base de datos independiente con el objetivo de incrementar la visibilidad, accesibilidad, reputación, uso e impacto de revistas en AA de calidad y revisadas por pares, independientemente de su disciplina, procedencia geográfica o idioma de publicación. Además, $D O A J$ muestra un fuerte compromiso con la transparencia de la información y las buenas prácticas en publicación académica. Al día de hoy, cuenta con más de 15000 revistas indexadas y vínculos con numerosas organizaciones, entre las que destacamos la presencia de Redalyc y Scielo (Scientific Electronic Library Online).

Más recientemente, a partir de un estudio que demuestra que, con la coordinación de esfuerzos de las principales organizaciones científicas del mundo, es posible una transición sostenible a escala global de un 
modelo de publicación científica cerrada a uno abierto (Schimmer, Geschuhn y Vogler, 2015), se crea el consorcio internacional de organizaciones de financiación de la investigación Coalition S. Este consorcio desarrolló lo que definió como Plan S, para que 2021 sea el año en el que la totalidad de la investigación financiada por sus integrantes sea puesta en disposición pública. Esto da cuenta de una modalidad de trabajo característica de los arcontes accesibles: la sinergia y la unión de esfuerzos entre Norte y Sur global a fin de potenciar los recursos de cada uno y sumar peso al movimiento hacia una mayor apertura (en un proceso cooperativo no libre de tensiones, ver nota 4). Otro ejemplo en ese sentido es la alianza entre la red de repositorios de AA latinoamericana La Referencia con OpenAIRE, proyecto europeo de infraestructura de acceso abierto para la investigación. Por el contrario, los arcontes corporativos se manejan bajo la lógica comercial de la fusión empresarial.

Incluso, en la $40^{\circ}$ reunión de la Conferencia General de la UNESCO, realizada en París en 2019, se resolvió, entre otras cuestiones, reconocer "la necesidad de contar con un nuevo instrumento normativo sobre la ciencia abierta, en forma de recomendación"; por ello, se le pide a la Directora General del organismo que organice una reunión intergubernamental en esta línea y que "adopte todas las medidas necesarias para garantizar un proceso de consulta inclusivo que conduzca a la elaboración de una recomendación sobre la ciencia abierta" (UNESCO, 2020, p. 33). A su vez, en el documento de trabajo presentado por el Comité Consultivo de la UNESCO (2019) que sirve de marco para la resolución presentada, se rescata en su Anexo II ejemplos a nivel global que se encaminan en el movimiento a una lógica arcóntica abierta. Entre los ejemplos enumerados, encontramos mencionado el Plan S, La Referencia, la infraestructura interinstitucional de comunicación para la publicación académica y la ciencia abierta AmeliCA (impulsada por UNESCO, CLACSO y Redalyc), ${ }^{4}$ la AOSP (African Open Science Platform) y la COAR (Confederation of Open Access Repositories), entre otros. El punto importante en este sentido es que el trabajo y esfuerzo desde la UNESCO se orienta a una recomendación en la línea de una lógica arcóntica abierta que funcione como instrumento legal sobre el que los estados miembros puedan desarrollar políticas en este sentido.

Por su parte, Scielo, iniciativa pionera de un modelo de publicación que busca indexar, preservar, mejorar y dar alta visibilidad a una colección de revistas evaluadas por pares, publicadas independientemente por sociedades científicas, asociaciones profesionales, universidades y otras instituciones de investigación (Packer, 2019), se encuentra en la implementación de un estándar de procedimientos para la inclusión de revistas en su colección. Dadas las características de esta biblioteca, que funciona como un consorcio de instituciones nacionales de investigación científica, se establecen ciertos requisitos básicos que son los mismos que comparten los arcontes corporativos (revisión por pares, periodicidad, relevancia temática, etc.). Un punto muy importante que diferencia esta lógica arcóntica es que especifica la obligatoriedad de que los materiales se encuentren disponibles en acceso abierto y promociona la alineación de las revistas con las "prácticas de comunicación de la ciencia abierta" (Scielo, 2018). Esta estrategia financiera y socialmente más sustentable para la difusión de las publicaciones científicas de las instituciones públicas y de sus comunidades de investigación fue adoptada también por otras bases de datos y repositorios institucionales, como Latindex, Redalyc o La Referencia (Aguado López y Vargas Arbeláez, 2016).

Asimismo, en el marco de una mayor accesibilidad al producto de la comunidad científica global, avanza cada vez de manera más consistente la política de autoarchivo que incluye la posibilidad de poner a disposición de la comunidad los que se conocen como preprints. Esto es, versiones borrador o versiones todavía "no revisadas por pares" para acelerar los tiempos de visibilización de los trabajos a la vez que ponerlos en discusión abiertamente de manera previa a su publicación formal en una revista. En esa línea, si bien se advierten ciertas cuestiones de cuidado, cada vez más revistas aceptan estos procedimientos. Así, varios repositorios de preprints están funcionando de manera muy intensa. Están puntualmente los casos de arXiv, bioRxiv, PeerJ, CogPrints y socArxiv que trabajan en colaboración con el Centro para la Ciencia Abierta. También Scielo habilitó en marzo de 2020 su servidor piloto de preprints como parte de su programa de 
cooperación internacional para el desarrollo del acceso abierto a la comunicación científica en todas las áreas de conocimiento.

Este movimiento de resistencia a la lógica corporativa encuentra sin embargo diversas maneras de reacción, es decir, el poder arcóntico hegemónico (ubicado en el Norte global) se está dando sus estrategias para no perder terreno ante el avance del acceso abierto. Por ejemplo, como puntualiza Lucas Luchilo (2018), en la promoción de oposiciones legales a prácticas de AA, con decisiones orientadas a entorpecer el depósito de artículos en repositorios institucionales, o con el desarrollo de grandes revistas de acceso abierto en el marco arcóntico corporativo que cobran altas tasas de procesamiento de artículos (APC, por su sigla en inglés, article processing charges). Es en este sentido en que empieza a desarrollarse el modelo híbrido de AA (la llamada vía dorada) que libera los contenidos, pero desplaza el origen de las ganancias desde las suscripciones para el acceso a los materiales al cobro por publicar ejercido sobre los autores. El costo, y, por ende, la apropiación de ganancias de la editorial, se transfiere del lector al autor. En vez de pagar por leer, se paga por ser publicado. Así, habida cuenta que las instituciones oficiales incorporan estas erogaciones como parte de su política de visibilidad científica, a partir de buscar garantizar la participación de los trabajos de sus académicos en las bases e índices de impacto dominante, vemos que este modelo llamado híbrido se sustenta fundamentalmente en dineros públicos. Entonces, al tiempo que las casas editoriales preservan su rol de arcontes corporativos y conservan el poder de controlar la cadena de publicación, archivo y acceso, la vía dorada continúa creando y resaltando las inequidades presentes entre países e instituciones (Guédon, 2015).

\section{CONSIDERACIONES FINALES}

En este trabajo hemos intentado dar cuenta de cómo, a través del tiempo, las tareas imprescindibles de búsqueda, identificación, recuperación, acceso y obtención física de las publicaciones científicas en tanto documentos no sólo se vieron influenciadas por cambios en los soportes tecnológicos, sino que también se convirtieron en una vía para generar lucro y dependencia a través de buscadores, bases de datos, catálogos, y sistemas de indexación. Más aún, al entender la evolución de la comunicación científica y la creciente necesidad de sistemas de protocolos, mecanismos de validación, prácticas de documentación y archivo, es posible analizar cómo estos elementos consolidan un determinado sistema científico dominante, a nivel global.

Los fines del poder arcóntico hegemónico son de dominación, y se combinan con las formas y lógicas impuestas para el ejercicio de una ciencia para pocos y en beneficio del capitalismo cognitivo. Opuesto a este dominio, en los últimos años han cobrado relevancia diferentes modalidades de publicación en AA, especialmente desde la región latinoamericana, donde la mayoría de las publicaciones se encuentran libremente disponibles en las propias revistas o en repositorios institucionales crecientemente integrados e interoperables. En una posición más radical, sitios web como Sci-Hub o LibGen enarbolan con orgullo la bandera de la piratería en una guerra donde sí se toman prisioneros, los papers, o más bien, se los libera de las ataduras de los arcontes comerciales. En este contexto, la emergencia de arcontes accesibles resulta una potencial transformación o nueva etapa por las que podría transcurrir la ciencia. Aunque estos cambios suelen llevar largos periodos, ciertos acontecimientos, que pueden parecer imperceptibles ante la potencia de las visiones dominantes, desencadenan procesos que ponen en tensión y cuestionan las formas "tradicionales" de producción, circulación y comunicación del conocimiento científico.

Sobre todo, es menester resaltar que la labor de los arcontes accesibles se convierte en una alternativa para la emancipación de una hegemonía instaurada. Consideramos que al menos tres fenómenos se convierten en temas de discusión: el primero, relacionado con las posibilidades de cuestionamiento e insumisión a las lógicas dominantes del sistema científico, la cienciometría, bibliometría y estándares de calidad y evaluación; el segundo, vinculado al desafío para universidades e instituciones de investigación como principales 
generadoras de la producción y circulación del conocimiento científico; y el tercero referido al beneficio social y de la comunidad científica a partir de prácticas que posibilitan procesos de apropiación del conocimiento.

El primero de estos puntos se enmarca en una discusión geopolítica más amplia, cuyo desarrollo excede los alcances de este trabajo, pero que no podemos dejar de mencionar. Así como los marcos teóricos y epistemológicos originados en el Norte Global obtienen automático reconocimiento como la ciencia que produce conocimiento universal (y universalmente aplicable), las propias condiciones institucionales y materiales de operación del campo científico a nivel global se establecen y se exportan como modelo único. Al respecto, existen desigualdades estructurales innegables entre disciplinas científicas y entre las instituciones ubicadas en distintos puntos del mundo. Sin embargo, como el campo científico internacionalizado deviene un "universo epistemológico compartido" y el investigador científico se concibe como un "profesional internacional” (Vessuri, 2013, p. 3), los valores del campo y la carrera científica operan de forma global.

Las prácticas archivísticas y de ejercicio de poder arcóntico no escapan a este fenómeno. Como se desprende de este artículo, los arcontes corporativos operan desde el Norte Global, mientras que regiones como América Latina se consolidan como las principales impulsoras de modelos accesibles, que paradójicamente también surgieron en aquellas tierras, pero aquí se han reconfigurado y adquirido perfiles contrahegemónicos. La fuerte adopción del acceso abierto en América Latina se ha planteado como una apuesta política que ha proporcionado mecanismos de reapropiación del conocimiento entendido como "un modelo de acción a favor de estructuras y mecanismos propios y pertinentes para la generación, la comunicación y el uso del conocimiento científico" (Aguado López y Vargas Arbeláez, 2016, p. 87). No obstante, tal como apuntan estos autores, para lograr el desarrollo adecuado y la expansión del AA en la región, persisten varios retos.

Por su parte, si las técnicas bibliométricas surgieron como herramientas para dar cuenta del movimiento de teorías, conceptos, métodos y herramientas; visualizar conexiones y colaboraciones entre redes de investigadores; y medir el impacto en tanto circulación de los artículos publicados (Vessuri, Guédon y Cetto, 2014), rápidamente se implementaron para la evaluación de individuos, instituciones, y países enteros. En paralelo con la mercantilización de la publicación científica, "un estilo de escritura, una lengua, un tipo de revista científica, y una forma de evaluación [...] fueron 'universalizados' a imagen y semejanza del modelo estadounidense de ciencia y de científico" (Beigel, 2017, p. 9). Actualmente, un puñado de arcontes decretan cuáles son las publicaciones significativas, es decir, aquellas de mayor calidad científica y mayor capital simbólico dentro de la ciencia mainstream. Tal lógica toma a la calificación e indexación como un hecho objetivo, y no como una forma particular y contingente de la métrica bajo una lógica de mercado, elaborada sin la participación de científicos periféricos (Beigel, 2017; Vessuri, Guédon y Cetto, 2014). Ante ello, vale la pena preguntarnos acerca de las condiciones que deben darse para que modelos regionales pudieran convertirse en estándares válidos y reconocidos por la ciencia global, y cómo abrir el campo de publicación y archivo sin quedar atrapado por una lógica comercial (Guédon, 2019).

El segundo punto que visualizamos como una posible línea a indagar en futuros trabajos, se relaciona con la capacidad institucional de, por un lado, enfrentar la comodificación del archivo y las publicaciones científicas, y, por otro, de impulsar otros modelos de arcontes para las ciencias. A tal fin, consideramos dos grupos de agentes como claves en la ruptura de la dependencia hacia los arcontes corporativos: universidades y centros de investigación, y Estados nacionales con sus divisiones dedicadas a la ciencia y la tecnología (ministerios, departamentos, secretarías, entre otros). Si los primeros son aquellos efectivos productores de conocimiento, los segundos son quienes delinean las políticas científicas que regulan la actividad. Sin embargo, la discutida autonomía del campo científico se opone, en ciertos casos, a la implementación de directrices provenientes de agentes externos. Irónicamente, los arcontes corporativos, guardianes e intérpretes de la circulación, publicación y conservación de la ciencia mainstream a nivel internacional, operan de acuerdo con lógicas de mercado que poco tienen que ver con los pretendidos valores tradicionales de la ciencia (al menos en su versión idealizada). 
En este sentido, consideramos que una mayor sinergia entre organismos públicos de ciencia y tecnología y las instituciones científicas sería fructífera en el avance hacia formas accesibles de publicación y archivo. A tal fin, las políticas científicas deberían contemplar explícitamente a la política editorial y archivística, un aspecto muchas veces invisibilizado ante otros urgentes reclamos de las comunidades científicas más vulnerables (aumentos salariales a investigadores, mayor cantidad de becas, subsidios y apoyo a la investigación, inversión en infraestructura y equipos, entre otros). Este aspecto es clave especialmente en contextos como América Latina, en donde la mayor parte de la investigación se desarrolla y financia, pero también se publica y archiva, con fondos públicos: los journals latinoamericanos dependen en gran parte de universidades e instituciones científicas estatales. Por supuesto, las acciones en pos de un acceso más abierto no son exclusivas del Sur Global: baste observar los resonantes casos de centros científicos de renombre que rescinden sus acuerdos con las grandes transnacionales editoriales o directamente llaman a su boicot. El ejemplo más reciente es la negativa del Massachusetts Institute of Technology (MIT) a renovar un contrato con Elsevier por no ajustarse con sus principios de publicación en acceso abierto (MIT Libraries, 2020).

El tercer punto que consideramos importante como eje de discusión tiene que ver con el carácter público de la ciencia. Podríamos acordar que dicha propiedad es adquirida en el momento en que se publica; sin embargo, las restricciones de acceso a los documentos físicos o digitales hacen que esta característica sólo se aplique bajo ciertas circunstancias. Si bien la condición de publicidad está determinada por distintos factores, es la noción de archivo y principalmente el tipo de arconte el que la podrá convertir en una posibilidad abierta o limitada. Es decir, ¿qué tan pública podrá ser la ciencia si sólo algunos miembros privilegiados de la comunidad científica pueden acceder a ella? Además, ¿es suficiente que un artículo sea publicado en una revista de acceso abierto para afirmar que es un bien público? ¿El acceso abierto apunta sólo a investigadores y entendidos? ¿Se piensa en la posibilidad de que la difusión del conocimiento científico pueda ser asimilada e interpretada por cualquier persona, teniendo en cuenta que para gran parte de la sociedad los centros de investigación continúan siendo "cajas negras"?

Es a partir de estos interrogantes que reflexionamos acerca de la idea de apropiación del conocimiento como una noción que merece ser puesta en discusión, ya que se hace presente en la modalidad de poder arcóntico corporativo entendida como la apropiación privada o comercial de ciencia. En la modalidad arcóntica accesible, el término cambia de sentido para significar tanto apropiación por parte de la comunidad científica como apropiación social de la ciencia.

Desde el modelo accesible, la apropiación se desprende de las prácticas contrahegemónicas al poder arcóntico corporativo impulsadas por los propios científicos y materializadas en modelos accesibles -lo que para Aguado López y Vargas Arbeláez (2016) sería la reapropiación; y de la misma idea de archivo, como acción que no remite al pasado, sino que concierne, ante todo, al mañana y a su promesa de significación futura. Es decir, la apropiación en este sentido no sólo implica el uso individual de la información acumulada y disponible por parte de los científicos, sino también responde a un principio organizador que tensiona el control del sistema dominante y permite nuevas interpretaciones. Por otro lado, el poder arcóntico accesible potencia el desarrollo de esa promesa abierta para la decodificación: los procesos de apropiación social de la ciencia, entendidos como aquellas prácticas que no sólo demandan participación en temáticas científicas, sino que ejercen una actuación directa y activa de movimientos sociales, vecinos organizados agrupaciones e individuos, basada en conocimientos e información para la resolución de un problema común.

La verdadera ciencia pública y publicada, la ciencia y el acceso abierto posibilitan el acceso social, pero también contribuyen al desorden y al exceso informacional. Es aquí donde la figura del arconte se hace imprescindible no sólo como guardián, sino verdadero intérprete. Quizá este arconte pueda utilizar la comunicación científica y la comunicación pública de la ciencia como herramientas para una verdadera democratización que potencie la apropiación crítica del conocimiento y quién sabe, agote el orden establecido por el capitalismo arcóntico actual. En tal sentido, para finalizar, consideramos que estos tres elementos de discusión deben ser establecidos no sólo como ámbitos de investigación que nos permitan conocer y entender 
cómo se producen y desarrollan los procesos, sino que deben formar parte de una agenda de discusión amplia en torno al rol de los Estados que, como responsables de políticas públicas en ciencia y tecnología, deben instrumentar acciones que permitan avanzar en alternativas al poder arcóntico corporativo.

\section{REFERENCIAS}

Aguado-López, E. y Vargas Arbeláez, E. J. (2016). Reapropiación del conocimiento y descolonización: el acceso abierto como proceso de acción política del sur. Revista colombiana de sociología, 39(2), 69-88. http://dx.doi.org/10.1 5446/rcs.v39n2.58966

Babini, D. (2019). Las ciencias sociales de América Latina y la oportunidad de contribuir con indicadores de evaluación. En M. Acero Cómez (Comp.), Sistemas de evaluación y edición universitaria (pp.105-136). Bogotá: Asociación de Editoriales Universitarias de Colombia.

Beigel, M. F. (2013a). Centros y periferias en la circulación internacional del conocimiento. Nueva sociedad, 245, 110-123. Recuperado de http://hdl.handle.net/11336/1232

Beigel, M. F. (2013b). David y Goliath. El sistema académico mundial y las perspectivas del conocimiento producido en la periferia. Pensamiento universitario, 5(15), 15-34. Recuperado de http://hdl.handle.net/11336/1313

Beigel, M. F. (2017). Científicos periféricos, entre Ariel y Caliban. Saberes institucionales y circuitos de consagración en Argentina: las publicaciones de los investigadores del CONICET. Dados: revista de ciências sociais, $60(3)$, 825-865. https://doi.org/10.1590/001152582017136

Björk, B. C. (2017). Gold, green, and black open access. Learned publishing, 30, 173-175. https://doi.org/10.1002 leap. 1096

Bourdieu, P. (1994). El campo científico. Redes, 1(2), 131-160.

Briceño, Y. (2013). El modo emergente de la comunicación de la ciencia: incidencias y gestión distribuida en América Latina (Tesis doctoral). Universidad de Los Andes, Facultad de Humanidades y Educación, Mérida. Recuperado de http://eprints.rclis.org/28500/

Darat, N. y Tello, A. M. (2016). "Desobediencia intelectual": resistencias a la privatización del conocimiento. POLIS: revista latinoamericana, 43. http://dx.doi.org/10.4067/S0718-65682016000100015

De Solla Price, D. J. (1965). Little science, big science. New York and London: Columbia University Press.

Debat, H. y Babini, D. (2020). Plan S en América Latina: Una nota de precaución. Revista CTS, 15(44), 279-292.

Derrida, J. (1997). Mal de archivo. Una impresión freudiana. Madrid: Editorial Trotta.

Fresco, A. (2013). Edición y Comunicación Cientifica: evolución y tendencias actuales (Tesis de maestría). Facultad de Humanidades, Comunicación y Documentación. Universidad Carlos III de Madrid, Madrid. Recuperado de $\underline{h}$ ttp://eprints.rclis.org/24471/1/Scholarly_comm_and_publishing_Evolution_and_Trends_AFresco.pdf

Foucault, M. (2002). La arqueología del saber. Buenos Aires: Siglo XXI.

Guédon, J. C. (1996). Electronic academic journals. From disciplines to seminars? En T. Harrison y T. Stephen (Eds.). Computer networking and scholarly communication in the twenty-first-century university (pp.335-350). Nueva York: State University of New York Press.

Guédon, J. C. (2015). Le libre accès: quo vadis? Ar(abes)ques, 79, 4-7. https://doi.org/10.35562/arabesques.799

Guédon, J. C. (2019). Plataformas (como Redalyc), revistas, libros y artículos digitales. ¿Cómo abrir el campo de cuestiones científicas sin quedar atrapado por una lógica comercial?. Palabra clave (La Plata), 8(2), e064. http s://doi.org/10.24215/18539912e 064

Hamel, R. E. (2013). El campo de las ciencias y la educación superior entre el monopolio del inglés y el plurilingüismo: elementos para una política del lenguaje en América Latina. Trabalhos em linguistica aplicada, 52(2), 321-384. https://doi.org/10.1590/S0103-18132013000200008

Himmelstein, D. S., Rodríguez Romero, A., Levernier, J. G., Munro, T. A., McLaughlin, S. R., Tzovaras, B. G., y Greene, C. S. (2018). Sci-Hub provides access to nearly all scholarly literature. eLife, 7, e32822. http://dx.doi .org/10.7554/eLife.32822 
Istratii, R., y Demeter, M. (2020). Plan S and the 'opening up' of scientific knowledge: a critical commentary. Decolonial subversions, 13-21.

Jinha, A. E. (2010). Article 50 million: An estimate of the number of scholarly articles in existence. Learnedpublishing. 23(3), 258-263. http://dx.doi.org/10.1087/20100308

Larivière, V.; Haustein, S. y Mongeon, P. (2015). The Oligopoly of academic publishers in the digital era. PLOS ONE, 10(6), e0127502. https://doi.org/10.1371/journal.pone.0127502

Luchilo, L. J. (2018). Revistas científicas: oligopolio y acceso abierto. Revista CTS, 14 (40), 41-79. Recuperado de ht tp://www.revistacts.net/files/Volumen 14 Numero 40/02Luchilo.pdf

Mendoza, S. y Paravic, T. (2006). Origen, clasificación y desafíos de las Revistas Científicas. Investigación y postgrado, 21(1), 49-75. Recuperado de https://www.redalyc.org/pdf/658/65821103.pdf

Merton, R. K. (1997 [1942]). La estructura normativa de la ciencia. Madrid: Editorial Alianza.

MIT Libraries. (11 de junio de 2020). MIT, guided by open access principles, ends Elsevier negotiations. MIT news. Recuperado de http://news.mit.edu/2020/guided-by-open-access-principles-mit-ends-elsevier-negotiations-0 $\underline{611}$

Moore, S. A. (2019). Revisiting "the 1990s debutante": Scholar-led publishing and the prehistory of the open access movement. Journal of the Association for Information Science and Technology, 71, 1-19. https://doi.org/10.100 2/asi.24306

Packer, A. L. (28 de febrero de 2014). SciELO Citation Index en el Web of Science. SciELO en perspectiva. Recuperado de https://blog.scielo.org/es/2014/02/28/scielo-citation-index-en-el-web-of-science/\#.XqYZHZlRfIU

Packer, A. L. (18 de junio de 2019). O modelo SciELO de publicação como política pública de acesso aberto. SciELO en perspectiva. Recuperado de https://blog.scielo.org/blog/2019/12/18/o-modelo-scielo-de-publicacao-comopolitica-publica-de-acesso-aberto/\#.XqY71JIRfIU

Polino, C. (2014). El proceso de autonomización de la ciencia moderna y la construcción del discurso divulgativo (siglos XVI y XVII). En Historia social y cultural de la divulgación. Módulo 1, Curso de Posgrado, Buenos Aires, Centro REDES.

Rossi, P. (2000). La nascita della scienza moderna in Europa. Roma/Bari: Laterza

Sabbatini, M. (1999). Evolución histórica de las publicaciones cientificas: de la République des Lettres hasta la World Wide Web (Tesis de maestría). Universidad de Salamanca, Salamanca. Recuperado de https://www.sabbatini. com/marcelo/artigos/1999sabbatini-republique.pdf

Schimmer, R., Geschuhn, K. K., y Vogler, A. (2015). Disrupting the subscription journals' business model for the necessary large-scale transformation to open access. Max Plank Digital Library Open Access Policy White Papers. http://dx.doi.org/10.17617/1.3

SciELO. (junio de 2018). Criterios, politica y procedimientos para la admisión y la permanencia de revistas cientificas en la Colección SciELO. Recuperado de https://wp.scielo.org/wp-content/uploads/Criterios-Rede-SciELO-es.pdf

Tello, A. M. (2018a). Una archivología (im)posible. Sobre la noción de archivo en el pensamiento filosófico. Síntesis: revista de filosofia, I(1), 43-65. http://dx.doi.org/10.15691/0718-5448Vol1Iss1a234

Tello, A. M. (2018b). Capitalismo arcóntico. En A. M. Tello, Anarchivismo: tecnologías políticas del archivo (pp. 219-256). Adrogué: La Cebra.

UNESCO. (2019). Estudio preliminar de los aspectos técnicos, financieros y jurídicos relativos a la conveniencia de contar con una recomendación de la UNESCO sobre la ciencia abierta. (40 C/63 + Add.). Conferencia General 40a reunión UNESCO. Recuperado de https://unesdoc.unesco.org/ark:/48223/pf0000370291 spa

UNESCO. (2020). Actas de la Conferencia General, 40a reunión, París, 12-27 de noviembre de 2019, volumen 1: resoluciones. (40 C/RESOLUTIONS VOL.1). Conferencia General 40ª reunión UNESCO. Recuperado de $\underline{h}$ ttps://unesdoc.unesco.org/ark:/48223/pf0000372579 spa

Vasen, F. y Lujano Vilchis, I. (2017). Sistemas nacionales de clasificación de revistas científicas en América Latina: tendencias recientes e implicaciones para la evaluación académica en ciencias sociales. Revista mexicana de ciencias politicas y Sociales, 62(231), 199-228. http://dx.doi.org/10.1016/S0185-1918(17)30043-0 
Verón, E. (1998). Entre la epistemología y la comunicación. CIC Cuadernos de Información y comunicación, 4, 149-155. Recuperado de https://revistas.ucm.es/index.php/CIYC/article/view/CIYC9899110149A

Vessuri, H. (2013). The dynamics of transformation processes in global social knowledge. En M. Kuhn y K. Okamoto (Eds.) Spatial social thoughts in global knowledge encounters (pp. 263-284). Stuttgart: Ibidem.

Vessuri, H., Guédon, J.-C., y Cetto, A. M. (2014). Excellence or quality? Impact of the current competition regime on science and scientific publishing in Latin America and its implications for development. Current sociology, 62(5), 647-665. http://dx.doi.org/10.1177/0011392113512839

\section{Notas}

1 Una línea alternativa sería pensar este fenómeno en el área de la tecnología, donde la operación de archivo característica es el patentamiento, que opera en el sentido opuesto a la publicación: patentar es delimitar y encerrar una porción de conocimiento (en el sentido práctico del saber-hacer) o una invención bajo determinado régimen de propiedad privada. El propio Robert Merton (1997 [1942]) colocaba a esta concepción de la propiedad intelectual en las antípodas del comunismo del ethos científico, considerándolos incompatibles. Sin embargo, bajo las modalidades de publicación académica a través de grupos editoriales, sujetas a una axiomática capitalista de producción y circulación de conocimiento, publicar-archivar también significa privatizar-apropiar-expoliar. Dado que una característica intrínseca de los bienes cognitivos es su multiplicabilidad y su reproducibilidad casi indefinida y a bajo costo, rasgos de los cuales de hecho depende el patrón de acumulación actual, surgen los distintos regímenes de propiedad intelectual y privatización del conocimiento como una forma artificial de generar escasez, privatizar, y por ende tornar, susceptibles de valoración y explotación a los bienes y servicios inmateriales (Darat y Tello, 2016).

2 Ver la obra de Fernanda Beigel (2013a, 2013b) para una discusión acerca de los circuitos de publicación científicoacadémica como instancias de consagración con capitales en juego a diferentes niveles. Gracias a las tecnologías digitales e informáticas, en las últimas décadas los archivos parecen haberse desterritorializado, pero, como bien pone de manifiesto la autora, la producción de conocimiento científico sigue bien geolocalizada en centros y periferias que exhiben marcadas diferencias estructurales y establecen relaciones crecientemente complejas, a pesar de que el poder arcóntico se extienda y ejerza de forma descentralizada y cada vez menos física.

3 Guédon (2015) destaca que lo que está en juego (el enjeu) en el movimiento del acceso abierto es precisamente la posibilidad de colocar a los científicos en el centro de un sistema de comunicación integrado a la cadena de trabajo propia de la investigación y controlado por las distintas comunidades disciplinares.

4 Si bien coinciden en los objetivos últimos del Plan S, tanto La Referencia como AmeliCA se han manifestado en contra de algunos de los puntos concretos del Plan, especialmente en el modelo de AA basado en el cobro de APC a los autores, por considerar que van en detrimento de los avances, conocimientos y esfuerzos acumulados desde el Sur Global, especialmente desde América Latina. Debat y Babini (2020) e Istratii y Demeter (2020), entre otros, han emitido críticas e introducido precauciones a la implementación global del Plan S. 